\title{
Author Correction: Using Bayes factor hypothesis testing in neuroscience to establish evidence of absence
}

\section{Christian Keysers (D), Valeria Gazzola and Eric-Jan Wagenmakers (D)}

Correction to: Nature Neuroscience https://doi.org/10.1038/s41593-020-0660-4, published online 29 June 2020.

In the version of this article initially published, a number of small errors occurred throughout the text, and several figures were out of order.

Figure 6 should have been Figure 3; Figure 3 should have been Figure 4; Figures 4 and 5 should have been Figs. 5 and 6 . Figure citations throughout the text have been updated to reflect this.

In the legend for Fig. $1 \mathrm{~b}$, the sentence beginning, "When an effect is present..." the last phrase should read "misses $\left(\mathrm{BF}_{+0}<1 / 3, \mathrm{red} \mathrm{bars}\right.$ and red percentages) are rare $(\mu=0.5)$ or absent $(\mu=1.2$ or 2$)$."

In Fig. 3a, the key for the graph on the right should have read, " $\mathrm{Max} \mathrm{BF}_{+0}: 0.99$ at $r=0.0$; Wide prior: $\mathrm{BF}_{0+}=4.5$; $\mathrm{Ult}_{\text {trawide }}$ prior: $\mathrm{BF}_{0+}=$ 6.1; User prior: $\mathrm{BF}_{0+}=8.4$ ". In the legend for Fig. 3a, the second-to-last sentence should read, "As can be seen, there is extreme evidence for $H_{1}$ in ShockObs, across all but the smallest priors (i.e., the gray, green and cyan dots all have $\mathrm{BF}_{+0}>160$ ), and there is moderate evi-

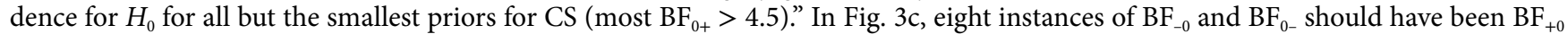
and $\mathrm{BF}_{0+}$, respectively.

In the paragraph beginning "The Bayesian approach..." the second sentence before the end of the paragraph should have read "If BF $\approx$ 1 we have absence of evidence."

In the paragraph beginning "Fig. 6 applies this logic...", the sentences beginning "Selecting 'Compare to..." should have read "Selecting

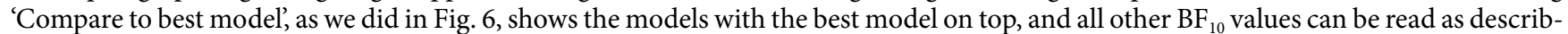
ing how likely that model is compared to the best model. If one selects 'Compare to null model', the null model is shown first, and all other $\mathrm{BF}_{10}$ values express likelihood relative to that null model."

In the paragraph beginning, "Selecting the option 'Prior..." the first sentence should have referred to dotted and blue lines.

The errors have been corrected in the PDF and HTML versions of this article.

Original
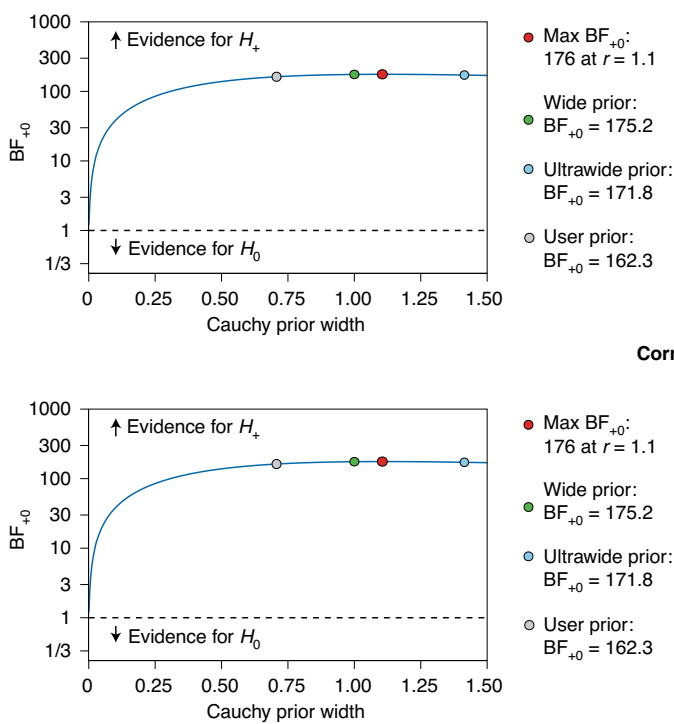

Max BF 176 at $r=1.1$

Wide prior:

$\mathrm{BF}_{+0}=175.2$

- Ultrawide prior:

$\mathrm{BF}_{+0}=171.8$

- User prior:

$\mathrm{BF}_{+0}=162.3$

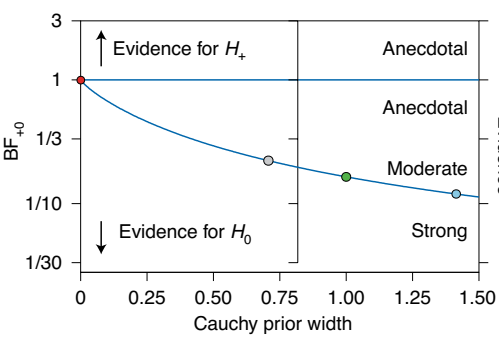

- Max BF 0.99 at $r=0.0$

- Wide prior: m $\mathrm{BF}+0=4.5$ - Ultrawide prior: $\mathrm{BF}+0=6.1$

- User prior: $\mathrm{BF}+0=8.4$

Corrected

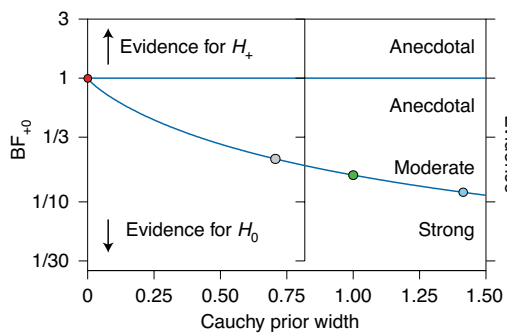

- Max BF 0.99 at $r=0.0$

- Wide prior: $\mathrm{BF}_{0+}=4.5$ Ultrawide prior: $\mathrm{BF}_{0+}=6.1$ - User prior: $\mathrm{BF}_{0+}=8.4$

Fig. 3 | Original and Corrected.

Published online: 6 October 2020

https://doi.org/10.1038/s41593-020-00710-7

(c) The Author(s), under exclusive licence to Springer Nature America, Inc. 2020 